\title{
Primeras impresiones sobre una Actividad de Aprendizaje Experiencial aplicada al desarrollo de nuevos productos.
}

\author{
Ángel Peiró-Signes ${ }^{\mathrm{a}}$, Marival Segarra-Oña ${ }^{\mathrm{b}}$, María de Miguel-Molina ${ }^{\mathrm{c}}$, Blanca de \\ Miguel-Molina $^{\mathrm{d}}$ \\ aDepartamento de Organización de Empresas (Universitat Politècnica de València, \\ anpeisig@omp.upv.es), bDepartamento de Organización de Empresas (Universitat Politècnica de \\ València, maseo@omp.upv.es), ${ }^{c}$ Departamento de Organización de Empresas (Universitat Politècnica \\ de València, mademi@omp.upv.es) y ${ }^{a}$ Departamento de Organización de Empresas (Universitat \\ Politècnica de València, bedemigu@omp.upv.es)
}

\begin{abstract}
In this paper we describe the results of the first application and the proposed adaptations for the use of an existing Experimental Learning Activity (ELA). Using the experience developed by Joe Tidd and John Bessant "New Product Development Game" which is oriented to illustrate the students some concepts related to the development of new products, we propose small improvements to guide the action of the students and to reach the activity objectives. The results of the activity are encoraging regardless the difficulties we encountered. The students evaluation of the activity indicated a high motivation towards the activity and also a high perception of the utility of the activity.
\end{abstract}

Keywords: Experiential Learning Activities, New product development, transversal competences.

\begin{abstract}
Resumen
En este trabajo describimos el resultado de la primera aplicación y las adaptaciones propuestas para utilizar una Actividad de Aprendizaje Experimental (AAE) existente. A partir de la experiencia desarrollada por Joe Tidd and John Bessant "New product development game" orientada a ilustrar a los estudiantes algunos conceptos relacionados con el Desarrollo de Nuevos Productos proponemos pequeñas mejoras para orientar la acción de los alumnos y conseguir los objetivos propuestos en la actividad. Los resultados de la actividad son esperanzadores a pesar de algunas dificultades encontradas. La evaluación de la actividad por los alumnos participantes indica una motivación alta hacia la actividad y una percepción de la utilidad de la actividad también elevada.
\end{abstract}

Palabras clave: Actividad de Aprendizaje experiencial, Desarrollo de nuevos productos, competencias transversales. 


\section{Introducción}

A la hora de planificar una asignatura a partir de los objetivos de aprendizaje y las competencias que pretendemos que adquieran los alumnos establecemos los contenidos, actividades y metodologías que pensamos más adecuadas en cada caso. El éxito depende en gran medida del grado de participación y la motivación del alumnado. De hecho, uno de los aspectos más complicados de la tarea docente consiste en motivar o, al menos, no desmotivar al alumno mientras se consiguen los objetivos de aprendizaje esperados.

Las estrategias que utilizan los profesores en este sentido son variadas y pasan normalmente por el aumento de la participación y responsabilidad del alumno en el aula. Las Actividades de Aprendizaje Experiencial (AAE) han demostrado en distintos niveles educativos (PeiroSignes, 2015) su capacidad y eficacia a la hora de dinamizar el trabajo del aula (PeiroSignes and Segarra-Oña, 2015).

LaForge y Busing (1998), indicaron los pasos a seguir para el desarrollo de una AAE planificación, introducción, ejecución de la actividad y retroalimentación. La mayor dificultad a la que se enfrenta el profesor se produce a la hora de planificar. Debe diseñar una actividad que sin llegar a la complejidad del mundo real refleje de forma relevante los aspectos importantes que se quieren desarrollar durante el curso (Halpern y Hakel, 2003). Adicionalmente, la actividad debe poner a los estudiantes frente a distintas situaciones que requieran de la toma de decisiones.

Sin embargo, es en la fase de retroalimentación, al final de la experiencia, donde se busca un proceso de reflexión sobre aquellos aspectos que han aflorado durante la actividad. Esta reflexión crítica es la que permite conseguir un aprendizaje significativo (Kolb, 1984; Wheeler y McLeod, 2002).

Por otro lado, las Actividades de Aprendizaje Experiencial permiten, de forma paralela, trabajar y reflexionar sobre la actuación de los alumnos a la hora de utilizar competencias de tipo transversal como: la comunicación, el trabajo en equipo o el liderazgo. Estas competencias son hoy de gran importancia para el desarrollo profesional de nuestros graduados.

\section{Objetivos}

En este trabajo describimos el resultado de la primera aplicación y las adaptaciones propuestas para utilizar una Actividad de Aprendizaje Experimental (ELA) existente. A partir de la experiencia desarrollada por Joe Tidd and John Bessant (2015) "New product development game", orientada a ilustrar a los estudiantes algunos conceptos relacionados con el Desarrollo de Nuevos Productos, proponemos pequeñas mejoras para orientar la acción de los alumnos y conseguir los objetivos propuestos en la actividad. "New product development game" es una actividad flexible diseñada para ilustrar algunos de los conceptos clave en el desarrollo de nuevos productos o servicios. Permite su adaptación y ampliación de forma que puede servir tanto como punto de partida para una sesión de clase magistral, como para su utilización más profunda evaluando las interrelaciones existentes

(cc) EY-NC-ND 2017, Universitat Politècnica de València 
entre diversos actores involucrados en el desarrollo de nuevos productos. La actividad original se puede escalar en función del grado de complejidad que deseemos introducir en el proceso.

En este artículo pretendemos exponer las primeras impresiones y resultados obtenidos de la actividad, evaluar los puntos de mejora y formular propuestas para en el futuro conseguir un mayor ajuste a los objetivos propuestos en la actividad.

\section{Desarrollo de la innovación}

Utilizamos una actividad tal y como originalmente fue diseñada por Joe Tidd and John Bessant. La actividad se inicia partiendo el grupo en varios equipos que van a competir entre ellos. A todos los alumnos se les indica que estarán involucrados en el diseño y producción de un nuevo producto y que es una competición. En el trabajo original se propone que el cliente sea el propio profesor y se les indica a los alumnos que deben de satisfacerle con su producto final.

Dentro de cada grupo, los alumnos se distribuyen según tres roles: Producción, Diseño y Observadores. Para el rol de Diseño se asignan 4 o más personas, 1 o 2 para el de Producción y, al menos, una desempeña el papel de observador.

A los alumnos que forman parte de producción se les envía a otra aula cercana, se les dan las instrucciones relacionadas con su tarea y se les indica que deben esperar allí. Posteriormente, se asignan distintas funciones dentro del equipo de diseño: Líder del proyecto (1 persona), responsable de Marketing (1 persona), responsable de I+D (1 persona) y Diseñadores (todos los demás).

Cada uno de ellos recibe una hoja informativa con el rol que debe de jugar, se reparten los materiales previamente preparados para la fase de diseño y se dan las instrucciones de inicio. Finalmente, fuera del aula donde se encuentra el equipo de diseño, se informa a los observadores de su rol como observadores de las dinámicas, comunicación y coordinación, de cada grupo.

Cada grupo debe diseñar un prototipo de coche autopropulsado en 30 minutos. Este prototipo deberá ser replicado por los operarios de Producción en 5 minutos. Finalmente, el cliente (profesor) realizará una evaluación del coche fabricado en relación a tres factores: cumplimiento de las expectativas del cliente, cumplimiento de los requisitos normativos establecidos en el juego y distancia recorrida por el vehículo autopropulsado.

La actividad está diseñada para establecer varias "trampas" en las que los grupos caigan y permitan reflexionar posteriormente en la fase de discusión y debate. Cada una de estas trampas se corresponde con un problema típico en el desarrollo de nuevos productos o servicios. El más que probable fracaso de los equipos nos permite explorar temas clave que deben de tenerse en cuenta a la hora de desarrollar nuevos productos.

En primer lugar, la deliberada separación de los equipos de producción y diseño permite trabajar aspectos de falta de comunicación o de involucramiento de los distintos 
departamentos desde el inicio del proceso o el diseño orientado a la fabricación. Por otra parte, nos permite ver la reacción de algunos alumnos ante la falta de información completa de lo que está ocurriendo en el proceso. En este sentido, los grupos Producción y Diseño disponen de información complementaria que es útil para el proceso de diseño y de producción. Esta información, al no establecer un canal de comunicación se pierde en el proceso. A pesar de no haber instruido al equipo de Diseño sobre ninguna limitación en cuanto a la comunicación con Producción, los equipos de Diseño no acuden al aula donde se encuentra el equipo de Producción a preguntar que tareas tienen asignadas o de que información disponen que pueda ser relevante. Tampoco los invitan a participar en el proceso de diseño, ya que se encuentran centrados exclusivamente en su tarea.

En segundo lugar, el equipo de diseño se pone rápidamente a diseñar, olvidando en muchos casos quién es el cliente. Aunque el profesor da una indicaciones sobre lo que pretende, un coche autopropulsado, es necesario entender de forma más profunda las necesidades que hay que satisfacer del cliente. Cuando los alumnos preguntan sobre dichas especificaciones, el instructor les ofrece un estudio de mercado. El estudio de mercado refleja unos requisitos distintos en cuanto a color y configuración a las que realmente quiere el cliente, el profesor. De esta forma se resalta otro punto clave a la hora del éxito en el Desarrollo de Nuevos Productos, la participación del cliente desde el inicio y a lo largo de todo el proyecto.

Por último, debido al escaso tiempo disponible y a la falta de implicación de producción en la fase de diseño, es difícil comprender los detalles de las ideas que el equipo de diseño trata de comunicar al Departamento de Producción para la fabricación del modelo definitivo. A esta falta de entendimiento se une una cierta desmotivación en el equipo de Diseño cuando ven las dificultades adicionales que el equipo de Producción tiene por las restricciones tecnológicas en el uso de algunos materiales.

\section{Resultados}

La actividad se llevó a cabo con estudiantes de nivel Master. La sesión de clase semanal consta de 2 horas. La actividad planificada estaba prevista para realizarse aproximadamente en una hora y quince minutos. Se inició la actividad tras los primeros 45 minutos de sesión, distribuyendo a los alumnos en 2 equipos con 7 componentes y uno con 8 ( 1 observador, $4 / 5$ en el equipo de diseño y 2 alumnos en producción). El primer análisis nos permite proponer una serie de cambios para futuras ediciones que mejoran de forma previsible los resultados obtenidos.

En primer lugar, en la sesión prácticamente todos los equipos pidieron una extensión en el tiempo de diseño, lo que impidió que la actividad se completara en el tiempo inicialmente estimado. De hecho, la discusión y retroalimentación de la actividad se tuvo que realizar en la siguiente sesión. Esto nos lleva a proponer que la actividad debe de plantearse desde el inicio de la sesión y debe dejarse un margen importante ante las eventualidades que pudieran concurrir. Estimamos que la próxima realización debería de disponer del tiempo completo de una sesión semanal ( 2 horas). En caso de finalización anticipada el instructor

(c)) BY-NC-ND 2017, Universitat Politècnica de València 
puede incidir brevemente, después de la fase de discusión, sobre los aspectos teóricos que se han experimentado en la actividad.

En segundo lugar, los equipos de producción en un aula aparte se encuentran sin saber cómo deben de actuar, desconocen completamente que ocurre la otra aula y no tienen que hacer nada básicamente durante el tiempo que dura el diseño. En este sentido, propondríamos dotar a estos equipos con algunos de los materiales de la actividad para que puedan experimentar con ellos. Por ejemplo, producción conoce las restricciones en el manejo de algunos de los materiales como los productos amarillos que deben manipularse con unos guantes de jardinería que dificultan enormemente el proceso. Dotar de estos materiales a los equipos de Producción mantendría la sensación la falta de comunicación con diseño a la vez que incrementaría su interés por la actividad.

En tercer lugar, los alumnos rápidamente preguntaron al cliente (el profesor) sobre los requerimientos del producto, para facilitar que los alumnos se centraran en las tendencias del mercado y no en el resto, el profesor ofreció rápidamente el estudio de mercado. En este sentido, dentro de las instrucciones generales y especialmente de las de la persona responsable de marketing debería de incorporarse la posibilidad y el coste de adquisición de un estudio de mercado. En este sentido el estudio de mercado debe tener un precio muy atractivo para incitar a los alumnos a caer en la segunda "trampa" preparada en la actividad.

En cuarto y último lugar, a pesar de que la gran mayoría de alumnos de la experiencia tenían una formación ingenieril, ninguno de los equipos llegó a completar dentro del tiempo esperado un prototipo viable para la prueba de distancia. Este aspecto es importante de cara a la satisfacción del alumno tras la experiencia. Las trampas nos permitirán forzar las situaciones de debate que deseamos de la experiencia pero es importante que los alumnos extraigan aspectos positivos sobre lo que han hecho. Además, esta situación también provoca que el impacto del diseño en el coste sea irrelevante, lo que impide que se pueda llevar a cabo una discusión tras la experiencia sobre estos aspectos. En este sentido, vistas las dinámicas en los distintos equipos y el uso que se dio a cada uno de los materiales, se propone limitar el uso de algunos de ellos a determinadas partes del vehículo. De esta forma los alumnos pueden llegar con mayor facilidad a un prototipo viable para posteriormente fabricarlo y probarlo. Por ejemplo, se puede limitar el uso de las cartas a las ruedas, el uso del cartón grande al chasis y los colores a la pintura del vehículo. Estas restricciones centrarán más la labor del equipo de diseño e incrementarán de forma notable las posibilidades de finalizar la etapa de diseño del prototipo. Además, como consecuencia, podremos evaluar el coste de cada prototipo y extraer conclusiones al respecto.

Por otro lado, se pasó una encuesta sobre aspectos relevantes relacionados con la motivación, utilidad y aspectos sobre como afrontaron los alumnos la actividad. Los resultados relativos a la motivación y percepción de aprendizaje son esperanzadores. Las medias sobre las preguntas contestadas por los 22 alumnos participantes en la actividad indican una motivación alta hacia la actividad y una percepción de la utilidad de la actividad también elevada. 


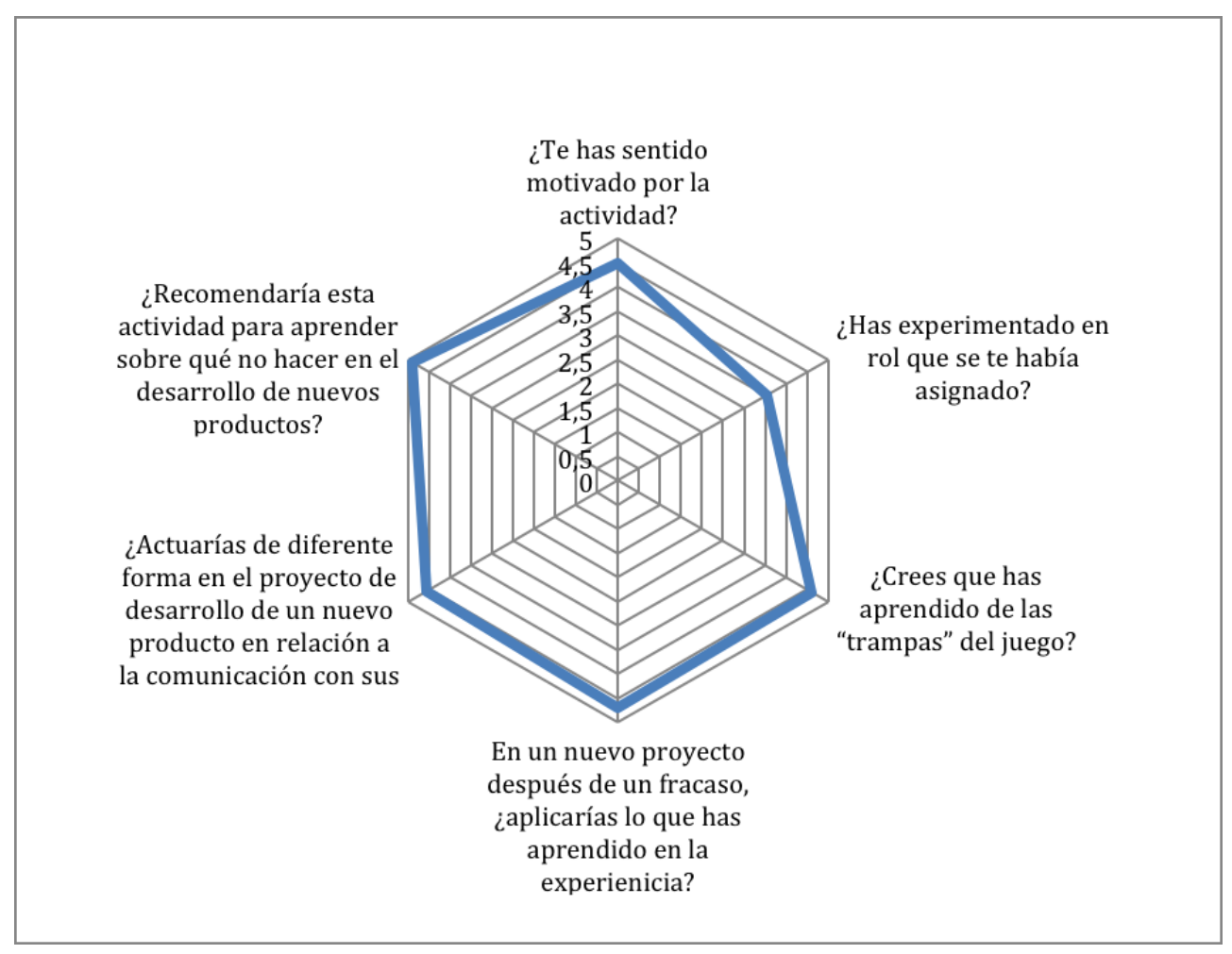

Fig. 1 Promedios de las preguntas de evaluación del uso de la AAE. Fuente: elaboración propia.

\section{Conclusiones}

Este artículo presenta los resultados preliminares de una actividad de aprendizaje experiencial diseñada para la enseñanza de los conceptos y habilidades necesarias el desarrollo de nuevos productos. La actividad pretende adentrar a los alumnos en varias "trampas" que pueden limitar de forma importante el éxito a la hora de desarrollar un nuevo producto. Tras la experiencia los alumnos deben apreciar la importancia que tiene la incorporación temprana de clientes y de otros departamentos implicados en desarrollo de los productos, como por ejemplo el Departamento de Producción. Por otro lado, deben poder evaluar el gran impacto que tiene la comunicación o la falta de ella en el proceso de desarrollo de nuevos productos.

Estos resultados, con las propuestas de mejora planteadas y la experiencia en la gestión de la actividad adquirida nos motiva como profesores a incorporarla dentro de las actividades de la asignatura. Futuras ediciones de la actividad aportarán nueva información relevante que permitirá realizar nuevos ajustes para mantener una actitud de mejor continua de nuestra labor docente. 


\section{Agradecimientos}

Este trabajo forma parte del estudio desarrollado por el Grupo de Aprendizaje Experiencial (GAE) creado como EICE en la Universidad Politécnica de Valencia (UPV). Los autores desean agradecer a la UPV por el apoyo a través del PIME 2017 "Adaptación y desarrollo de aprendizajes experienciales al contexto de las asignaturas".

\section{Referencias}

TIDD, J. y BESSANT, J. (2015). New Product Development Game. <http://www.innovation--portal.info/> [Consulta: 5 de enero 2017] John Wiley and Sons Ltd.

HALPERN, D. y HAKEL, M. (2003). “Applying the science of learning”. Change, vol. 35, issue 4, p. $36-41$.

KOLB, D. (1984). Experiential learning: Experience as the source of learning and development. Englewood Cliffs, NJ: Prentice Hall.

LAFORGE, R. y BUSING, M. (1998). "The use of industrial software to create experiential learning activities in operations management courses". Production and Operations Management, vol. 7, issue 3, p. 325-334.

MEYERS, C. (1986). Teaching students to think critically. San Francisco, CA: Jossey-Bass.

PEIRO-SIGNES, A., SEGARRA-OÑA, M., DE MIGUEL-MOLINA, M., J. ALBORS-GARRIGÓS, J. y DE-MIGUEL-MOLINA B. (2015). "The experiential learning activity: benefits and difficulties in real learning environments". Proceedings of ICERI2015 Conference, p. 7231-7237.

PEIRO-SIGNES, A. y SEGARRA-OÑA, M.; (2015). "Experiential learning as a dynamizer of class activity”. Proceedings of ICERI2015 Conference, p. 1010-1013.

SMITH, G. (2003). "Beyond critical thinking and decision making: Teaching business students how to think". Journal of Management Education, vol. 27, issue 1, p. 24-51.

WHEELER, J., y MCLEOD, P. (2002). "Expanding our teaching effectiveness: Understanding our responses to in-the-moment classroom events". Journal of Management Education, vol. 26, issue 6, p. 693-716. 